\title{
Editorial
}

\section{THE PUZZLE OF CHRONIC PAIN : WILL GENETICS FORCE A MAJOR BREAKTHROUGH IN THE PATHOPHYSIOLOGY AND THE TREATMENT OF CHRONIC PAIN?}

\author{
J. E. R. Devulder \\ Key words: chronic pain, pain models, genetic polymorphism
}

Since the inauguration of the International Association for the Study of Pain (IASP) in 1973, chronic pain has received more attention. Pain was initially considered as a symptom, whereas currently, the European Federations of International IASP Chapters (EFIC) consider chronic pain as a disease and propagate that pain patients should not longer suffer in silence. The understanding of the pathophysiology of pain has progressed and animal models, cell culture studies and even human cognitive behavioural experiments have been constructed to mimic pain in humans. Unfortunately, the more is known about chronic pain, the more complex the syndrome becomes and many patients still cannot be cured. This paradox asks for an explanation, not in the least because new pain syndromes have appeared such as fibromyalgia , chronic fatigue syndrome, complex regional pain syndrome and the failed back surgery syndrome. Despite that research has shown variable tissue reactions to pain in both humans and other species, a specific patientoriented pain treatment, targeting specific deficits or physiological mechanisms is still lacking.

Address for correspondence:

Jacques E R Devulder MD, PhD

University Hospital, Ghent

Department of Anaesthesia

Section Pain Clinic

De Pintelaan 185

9000 Ghent

Belgium

Tel: +3292403018

Fax: +329240 3835

E-mail: jacques.devulder@ugent.be
Could genetic mapping become an adjuvant investigative tool to explain pain and target a structured treatment, or will it make the pathophysiology of chronic pain even more complex? Chronic pain can be considered as 'a biomedical problem' but more and more scientist are proponents of the 'biopsychosocial model' approach.

Some physicians take chronic pain only seriously if it is documented by clinical signs. Pain as a symptom is considered either as having a biological origin, or as a psychological trait, if no clear diagnosis can be made or if the complaints are not consistent with the diagnostic abnormalities. Science has progressed and sensitization has been described with sometimes a transition from acute to chronic pain. (1). Many physiological systems influence the peripheral and central nervous system leading to changes in expression of ion channels, receptor types and different excitatory and/or inhibitory neurotransmitters. Specific drugs try to inhibit those modulations. Sometimes they alleviate pain, but in many patients they are inadequate. This can be partly attributed to changes in receptor or ion channel expression, although new mechanisms unravelling the puzzle are becoming apparent(2). Particular molecules and proteins formed by pain signals such as mitogen-activated kinases, interleukines and many others, initiate a loop of enzyme processes, leading to genetic transcription changes (transcriptome changes) and protein formation (proteomes)(3). Some genes, after having remained silent for a long period, become active and initiate new receptors, channels or transmitters; i.e. the tetrodoxin-sensitive sodium channels that 
are replaced by tetrodotoxin-resistant sodium channels, sometimes leading to facilitation of pain signals (2). Pain inhibition becomes increasingly difficult because the novel ion channels have different reaction characteristics and may become resistant to classical channel blockers. Nerve fibres involved in pain transmission, become more sensitive and intensified pain signals reach the central nervous system (1).

Often, the N-methyl-dextro- aspartate (NMDA) receptor, specific of the excitatory transmitter glutamate, plays a pivotal role, but specific receptor blockers without side-effects are not available (4). Ketamine, a specific anaesthetic agent with psychotropic side effects is still the principal drug for blocking those receptors (5). The drug blocks only one receptor type and is not useful for all types of pain. Even small doses, have potential side-effects. Other drugs such as paracetamol, non-steroidal anti-inflammatory drugs (NSAIDs) and opioids, are available but their harmful effects sometimes outweigh the benefits. Anticonvulsants and tricyclic antidepressants have the potential to block some ion channels or to interfere with excitatory or inhibitory neurotransmitters. However, their cognitive side-effects cannot be ignored (6). As mentioned earlier drugs sometimes help to alleviate pain, but success is by no means guaranteed.

IASP has stressed the importance of considering chronic pain as a biopsychosocial model and of approaching it in a multidisciplinary fashion. The patients' cultural background, social living conditions and psychological structure can indeed enhance the pain experience (7). This experience can incite a negative circle. The "fear avoidance model" proposed by Roelofs et al is a useful model demonstrating devastating results of behavioural influence (8). Anxious people are prone to develop chronic pain. Because of noxious stimuli, they fear movement, resulting in a reduction of their performance level. Those patients experience more pain in the skin and tissues. Psychological factors play an important role and patients sometimes need to learn to live with their dysfuncions while listening to their body and to adapt their activities. A cognitive behavioural approach can have an important positive impact on their pain cognitions and prevent catastrophizing (9). Most research to pain therapy and psychology is based on the cognitive behavioural approach. The way patients think about their pain, feel it and cope with it, is the cornerstone of this psychological approach (10). Although cognitive behavioural therapy looks very apprehensive, some patients learn to manage their pain better. Yet, elimination of pain by this technique is rare.
Anyway, an approach that combines physical therapy with psychotherapy requires a patient's motivated input. Defensive attitudes, drug misuse and abuse can perpetuate a continuous pain behaviour. Treating patients in this way sometimes only leads to psychosocial "acceptance" of pain because of biomedical failure (11).

Are those patients deemed to have pain for the rest of their life?

Better insight into the human and animal genome by medical genetic mapping gives clinicians hope for a better understanding of disease and future treatment options (3). Genetic research has a long history but major progress was particularly made in the last decade. In the early years of human genetic history, genetic information was thought to flow into one direction, from DNA to RNA and protein production (12). Currently we know that the inverse can occur because proteins can affect the reading of DNA(3). They permit cells to serve as drug factories and they may enable the correction of genetic errors that predispose to pain. This can be very important because genetic methods may allow therapeutic agents to interact with DNA or mRNA instead of with proteins.

Some genes predispose individuals to specific conditions, the primary symptom of which is pain; other genes render individuals more susceptible to disease entities that may damage nerves and other tissues, and secondarily lead to chronic pain. These include diabetes mellitus, hereditary demyelinating neuropathies and cancer.

Currently researchers have found some painsusceptible genes coding for pain-relevant proteins, either excitatory or inhibitory (13). They have also found small allelic variations in subtle nucleotide sequences: polymorphisms. The latter finding seems to be very important because a more ambitious project than merely drafting the sequence of the human genome is under way, notably the development of a human haplotype map (HapMap), designed to create a catalogue of common allelic variations, even combinations of different variants associated with human pain traits (14).

Allelic differences can include a significant portion of different nucleotide bases. However, far more commonly, an error in one single nucleotide " a single nucleotide polymorphisms" or "SNP", occurs (15). It might result in genetic transcription associated with protein formation with consequences such as functional loss, or only subtle functional consequences or protein formation in higher or lower amounts Mostly, these polymorphisms not only affect one but different body systems. 
Zubieta et al. noticed that polymorphism on the COMT met val(sup158) had an important effect on pain. The met 158 allele of the catechol-Omethyltransferase (COMT) affects the metabolism of catecholamines, which also affects the modulation of responses to sustained pain in humans. It does not only affect dopaminergic, adrenergic /noradrenergic neurotransmission but has also a tremendous influence on the $\mu$ receptor functioning and on psychosocial wellbeing(13).

Their study demonstrated that a common functional genetic polymorphism of the human COMT enzyme causes unique regional neurochemical brain system activations paralleled by distinct psychophysical response traits during sustained pain. This could be visualized using PET scanning, and multidimensional pain questionnaires.

Polymorphisms in the GA118 allele of the OPRM1gene ( $\mu$ - opioid receptor gene) have important consequences for the plasma levels of morphine and morphine 6 glucuronide (16). This explains the differences in morphine effect and tolerance. Such variables might have major implications in the treatment of cancer and non-cancer pain.

Those SNP's have also been described for the OPRD1 subtype gene (responsible for dynorphine production) and for the TRPV 1 Val 585 allele producing the vanilloid receptor (nociceptor), responsible for nociceptive pain sensation (17). Opioids can cross biological membranes and target their specific opioid receptors by passive diffusion or can be actively transported by membrane transporter proteins. One of these transporters, the efflux transporter P-glycoprotein (P-gp), is important for opioid and other drugs such as digoxine and antiretrovirals. P-gp's function is to reduce intracellular drug accumulation through active efflux, thus reducing the effective drug level at the target sites. Studies using

P-gp knock out mice, or blocking expression of P-gP alters the brain-to-plasma ratio (18). Downregulation of P-gp expression with antisense reduces the brain-toblood transport of morphine and endorphins, enhancing morphine analgesia. Some opioids, such as hydromorphone and methadone, suppress P-gp activity while others such as fentanyl only inhibit part of its activity. $P$-gp is encoded in the ABCB1 gene. Several polymorphisms and mutations have been demonstrated, e.g. SNP C3435T in the ABCT1 gene. (19). A clearer insight into those systems might in the near future provide guidelines for opioid therapy.

Congenital indifference to pain in patients lacking some genetic structures, or having mutations in some parts of chromosome 1 (1q21-q22) has an impact on the TRKA receptor ( one of the nerve growth factor receptors), which is important to the ongoing pain signalling cascade (20). Currently, different polymorphisms are described (HSAN type I-V ; hereditary sensory and autonomic neuropathies), all with different phenotypical changes but pain insensitivity is the main symptom (21). The defect (HSANs) is often associated with different pathological conditions such as autonomic dysfunctions or mental retardations.

\section{CONCLUSION}

Chronic pain remains a burden to society, and is still difficult to treat. Despite major advances in the diagnosis by psychosocial screening and better visualisation techniques such as brain mapping by functional MRI or PET scanning, the reaction of the human body is not well understood. The understanding and mapping of different pain-susceptible genes seem to be progressing and will clarify why some patients do not feel pain, or do not respond to medication. Although the technique looks promising, the question still remains if genetic mapping will constitute a breakthrough in chronic pain treatment. Chronic pain is so complex that its pathophysiology and behavioural ramifications could evade simple dissection even by advanced genetic technologies. Probably genetics will be of added value but will not force a major therapeutic breakthrough in the nearby future.

\section{REFERENCES}

1. Van Wilgen C, Keizer D. The sensitization model: method to explain chronic pain to a patient. Ned Tijdschr Geneeskd, 2004; 148:2535-8

2. Gold MS, Weinreich D, Kim CS, Wang R, Treanor J, Porreca F et al. Redistribution of Nav1.8 in uninjured axons enables neuropathic pain. J Neurosci, 2003; 23:158-66

3. The genetics of pain $p 4$ IASP press Seattle 2004 editor Jeffrey Mogil

4. Willert RP, Woolf CJ, Hobson AR, Delaney C, Thompson DG, Aziz $\mathrm{Q}$. The development and maintenance of human visceral pain hypersensivity is dependant on the NMDA receptor. Gastroenterology, 2004 Mar;126(3):683-92.

5. Jorum E, Warncke T, Stubhaug A. Cold allodynia and hyperalgesia in neuropathic pain: the effect of NMDA receptor antagonist ketamine-a double blind,cross over comparison with alfentanil and placebo. Pain, 2003 Feb;101(3):229-35

6. Goucke C. The management of persistent pain. Med J Aust, 2003 May 5;178(9):444-7

7. Loeser JD. Pain and suffering. Clin J Pain, 2000 Jun;16(2 Suppl): S2-6 
8. Roelofs J, Peters ML, Fassaert T, Vlaeyen JW. The role of fear of movement and injury in selective attentional processing in patients with chronic low back pain: a dot-probe evaluation. J Pain. 2005 May;6(5):294-300

9. Severeijns R, van den Hout MA, Vlaeyen JW. The causal status of pain catastrophizing: an experimental test with healthy participants. Eur J Pain. 2005 Jun;9(3):257-65.

10. Lazarus AA, Abramovitz A. A multimodal behavioural approach to performance anxiety. J Clin Psych, 2004 Aug;60(8):831-40

11. Viane I, Crombez G, Eccleston C, Devulder J, De Corte W. Acceptance of the unpleasant reality of chronic pain: effects upon attention to pain and engagement with daily activities. Pain, 2004 Dec;112(3):282-8.

12. Watson J, Crick F. Molecular structure of nucleic acids. Nature, 1953 Apr 25; 171(4356): 737-8

13. Zubieta JK, Heitzeg MM, Smith YR, Bueller JA, Xu K, Xu Y et al. COMT val (sup158) met Genotype affects $\mu$-opioid neurotransmittor responses to a pain stressor. Science, 2003 Feb 21;299(5610):1240-3

14. Cardon L, Abecasis G. Using haplotype blocks to map human complex trait loci. Trends Cenet, 2003; Mar;19(3):135-40

15. Gardner S, Wagner M. Software for optimisation of SNP and PCR-RFLP genotyping to discriminate many genomes with the fewest assays. BMC Genomics. 2005 May 16;6(1):73
16. Lötsch J, Zimmermann M, Darimont J, Marx C. Dudziak R, Skarke $C$ et al. Does the A $118 \mathrm{G}$ polymorphism at the $\mu$ opioid receptor gene protect against morphine-6-glucuronide toxicity? Anesthesiology, 2002 Oct;97(4):814-9

17. Kim H, Neubert JK, San Miguel A, Xu K, Krishnaraju RK, Iadarola MJ et al. Genetic influence on variability in human acute experimental pain sensitivity associated with gender, ethnicity and psychological temperament. Pain, 2004 Jun; 109(3):488-96.

18. King M, Su W, Chang A, Zuckerman A, Pasternalk GW. Transport of opioids from the brain to the periphery by P-glycoprotein. Peripheral action of central drugs. Nat Neurosci, 2001 Mar;4(3): 268-74

19. Hoffmeyer S, Burk O, von Richter O, Arnold H, Broekmoller J, Johne A et al. Functional polymorphisms of the human multidrug resistance gene: multiple sequence variations and correlation of one allele with P-glycoproteine expression and activity in vivo. Proc Natl Acad Sci USA, 2000 Mar 28;97(7):3473-88

20. Mamet J, Lazdunski M, Voilley N. How nerve growth factor drives physiological and inflammatory expression of acid-sensing ion channel 3 in sensory neurons. J Biol Chem, 2003 Dec 5;278(49): 48907-13

21. Indo $Y$. Genetics of congenital insensitivity to pain with anhidrosis (CIPA) or hereditary sensory and autonomic neuropathy type IV. Clinical, biological and molecular aspects of mutations in TRKA(NTRK 1) gene encoding the receptor tyrosine kinase for nerve growth factor. Clin Auton Res. 2002 May:12 Suppl 1:120-32 